\title{
COVID-19: DISTANCIAMIENTO SOCIAL, SUS EFECTOS PSICOLÓGICOS Y 10 ESTRATEGIAS PARA SOBRELLEVARLO
}

\section{ACCOMPANIMENT OF STUDENTS WITH HEARING DISABILITIES AT THE USAC SCHOOL OF PSYCHOLOGICAL SCIENCES}

\section{Referencia del artículo}

Vides, R. (2020). COVID-19: Distanciamiento social, sus efectos psicológicos y 10 estrategias para sobrellevarlo. Revista Científica del SEP. 3(1), 17-23. DOI: https://doi.org/10.36958/sep.v3i01.27

\author{
Rory Rene Vides Alonzo \\ psiquism5to@yahoo.com \\ Médico y Cirujano, PhD Psicología Medica y Salud Mental \\ Centro Universitario de Oriente CUNORI \\ Universidad de SanCarlos de Guatemala
}

Eileen Mariemilia Alegria Barrow

Estudiante de la carrera de Médico y Cirujano

Centro Universitario de Oriente CUNORI

Universidad de SanCarlos de Guatemala

Isolina Janeth Morales Guerra

Estudiante de la carrera de Médico y Cirujano

Centro Universitario de Oriente CUNORI

Universidad de SanCarlos de Guatemala
Carlos Junior Sagastume Roque

Estudiante de la carrera de Médico y Cirujano

Centro Universitario de Oriente CUNORI

Universidad de SanCarlos de Guatemala

Juan Danilo Montejo Galdámez

Estudiante de la carrera de Médico y Cirujano

Centro Universitario de Oriente CUNORI

Universidad de SanCarlos de Guatemala

Fecha de recibido: 30 junio 2020

Fecha de aceptado: 23 octubre 2020

\section{RESUMEN}

El estado de alerta decretado por el gobierno para hacer frente a la crisis sanitaria en la que nos encontramos en relación a la expansión del coronavirus (COVID-19), obligó a cambios en los patrones de comportamiento y detenimiento del funcionamiento habitual del día a día. Cuando la situación dura más de unas pocas semanas, a menudo surgen problemas de salud relacionados con el aislamiento social.

El brote de la enfermedad por coronavirus 2019 (COVID-19) puede causar estrés en algunas personas, la ansiedad por la pandemia puede ser abrumadora y causar fuertes emociones en adultos y niños. En efecto, esta situación tiene una potente carga psicológica sobre nosotros.

Durante la pandemia, es razonable esperar que las enfermedades mentales se vean afectadas de diferentes formas por aislamiento prolongado. Los efectos más frecuentes son: Depresión paranoide, Esquizofrenia residual, Trastorno esquizoide de la personalidad, entre otras. Demasiada información puede provocarnos estrés, ansiedad u angustia que afecta nuestro estado emocional, que nos lleva a realizar diversas comprobaciones, como buscar información nueva y actualizada. Para mitigar estos factores estresores y sus posibles consecuencias, existen algunas estrategias que pueden utilizarse para prevenir y reducir los efectos del aislamiento.

\section{PALABRAS CLAVE}

Covid-19, enfermedad mental grave, estrés, depresión, aislamiento, distanciamiento social

\section{ABSTRACT}

The state of alert decreed by the government to face the health crisis in which we find ourselves in relation to the expansion of the coronavirus (COVID-19), forced changes in behavior patterns and stopping the usual operation of the day day. When the situation lasts for more than a few weeks, health problems related to social isolation often arise.

The outbreak of coronavirus disease 2019 (COVID-19) can cause stress in some people, anxiety about the pandemic can be overwhelming and cause strong emotions in adults and children. Indeed, this situation has a powerful psychological burden on us.

During the pandemic, it is reasonable to expect that mental illness will be affected in different ways by prolonged isolation. The most frequent effects are: Paranoid depression, Residual schizophrenia, Schizoid personality disorder, among others. Too much information can cause us stress, anxiety or anguish that affects our emotional state, which leads us to perform various checks, such as looking for new and updated information. To mitigate these stressors and their potential consequences, there are some strategies that can be used to prevent and reduce the effects of isolation.

\section{KEYWORDS}

Covid-19, serious mental illness, stress depression, isolation, social distancing 


\section{1.- INTRODUCCIÓN}

Luego de esta pandemia por COVID-19, otra podría seguirla rápidamente, la de las enfermedades mentales y del comportamiento.

Desde que se diagnosticó el primer caso de infección por coronavirus 2019 (COVID-19), se ha extendido por el mundo y ha impulsado la acción global.

El 13 de marzo del 2020, el primer caso en Guatemala, el día que vino a cambiar la vida de muchos guatemaltecos para siempre. Que ha traído esfuerzos sin precedentes para la población en general a practicar el distanciamiento físico (llamado en la mayoría de los casos "distanciamiento social") no solo a Guatemala, sino que, en países de todo el mundo, obligándonos así a cambios en los patrones de comportamiento y de nuestra rutina diaria habitual.

Cuando la situación dura más de unas pocas semanas, a menudo surgen problemas de salud relacionados con el aislamiento social. El distanciamiento de meses entre personas significa que las consecuencias potencialmente graves de una pandemia (recesión, desempleo, etc.) pueden provocar un aumento de los problemas de salud físicos y mentales.

Sin embargo, esta soledad y aislamiento pueden dañar la salud de todos los grupos de edad. Durante este período de distanciamiento social, a algunas personas les irá mejor que a otras según sus hábitos y comportamientos. Aunque la tecnología moderna se usa ampliamente, en las condiciones actuales, no puede reemplazar el contacto humano.

La pérdida de las actividades diarias normales, la reducción del contacto social y físico con los demás, a menudo muestra aburrimiento, frustración y una sensación de aislamiento del resto del mundo, preocupando así a la población. Esta frustración se ve agravada por la incapacidad de continuar con actividades diarias normales como ir de compras, satisfacer las necesidades básicas y participar en actividades comunitarias (ir a la iglesia o al parque).

¿Acaso, nuestro comportamiento y rasgos psicológicos pueden tener un impacto en la manera en la que nuestra sociedad responda a la crisis y afronte los desafíos venideros?

El brote de la enfermedad por coronavirus 2019 (COVID-19) puede causar estrés a algunas personas. La ansiedad por la enfermedad puede ser abrumadora y causar fuertes emociones en adultos y niños. Hacer frente al estrés lo fortalecerá a usted, a las personas importantes en su vida y a su comunidad. Al fortalecer la salud mental, podemos ser más fuertes que nunca.
El estrés durante un brote de enfermedades infecciosas puede incluir las siguientes reacciones:

- Preocupación y temor por su salud y la salud de sus familiares.

- Alteración en la alimentación y los patrones de sueño.

- Dificultad para conciliar el sueño o prestar atención.

- Exacerbar las enfermedades crónicas.

- Exacerbar los trastornos mentales.

- Incrementar el consumo de alcohol, tabaco u otras drogas.

Todos responden de manera diferente a situaciones estresantes. La forma en que responda al brote puede depender de su historial, en aspectos que lo diferencian de los demás y de la comunidad en donde vive.

El estrés de una crisis puede ser más fuerte en:

- Personas de la tercera edad y con enfermedades crónicas que tengan mayores factores de riesgo de enfermarse de forma grave a causa del COVID-19

- Niños y adolescentes

- Personas voluntarias que ayudan a combatir el COVID-19, como personal médico, proveedores de atención en salud y personal de respuesta a emergencias

- Pacientes con enfermedades mentales, incluidas las personas con trastornos por abuso de sustancias.

Los psiquiatras que ayudan a personas con enfermedades mentales graves necesitan información sobre vulnerabilidades y requisitos de tratamiento de esta población mientras transcurre la pandemia de COVID-19.

La respuesta a nivel mundial al COVID-19 debe extenderse al entorno de los síntomas en los pacientes, ya que los síntomas pueden alterar significativamente la respuesta de la población general, entre los principales síntomas de psicosis se pueden señalar los siguientes:

Paranoia. Las formas de comunicación a distancia pueden aumentar la paranoia de los pacientes, ya que se les exige que se comuniquen a través de herramientas electrónicas, por ejemplo; ver a su psiquiatra en una pantalla. El miedo experimentado por el personal lo sienten los pacientes cuyo pensamiento paranoico se puede engrandecer.

Delirios. Algunos pacientes han incorporado COVID-19 a sus antiguas creencias, como que los Illuminati tienen el control 
de la pandemia mundial o que la población mundial merece ser sancionada. Otro ejemplo podría ser un paciente que cree que es médico y que da consejos médicos erróneos sobre COVID-19 a otros pacientes de la unidad.

Alucinaciones. Las personas con enfermedad mental grave (EMG) pueden atribuir la información que reciben a sus "voces" o escuchar el ruido de los virus. Lo más importante es la necesidad de que el psiquiatra sea perceptivo al hecho de que las alucinaciones auditivas pueden interferir con la capacidad de comunicarse por teléfono. El paciente mezcla todas las voces, incluidas las del psiquiatra. La pérdida de señales visuales puede poner en grave peligro la forma de comunicación real que existe anteriormente entre el médico y el paciente.

Déficit cognitivo. Las personas con déficit cognitivo pueden no entender de qué se trata todo esto, lo que lleva a su incapacidad para comprender la gravedad de la situación. Es posible que no recuerden lo que les han enseñado sobre el virus y pueden requerir recordatorios varias veces al día para que adopten nuevos hábitos, como lavarse las manos con más frecuencia y practicar el distanciamiento social. Las personas con déficit cognitivos pueden ser incontinentes, lo que lleva a los cuidadores a tener contacto físico con el individuo varias veces al día, lo que empeora si los pacientes presentan agitación o agresividad.

Desorganización. Al igual que aquellos con déficits cognitivos, los pacientes que no tienen organización pueden tener dificultades para seguir los procedimientos sobre higiene de manos y distanciamiento social. También pueden estar confundidos acerca de su estadía en el hospital o por qué no pueden recibir visitas.

Ansiedad. Los pacientes con síntomas de trauma previos o trastorno de estrés postraumático (TEPT), especialmente TEPT complejo, pueden ser provocados por miedos sobre COVID-19: "El hospital ya no es un lugar de confianza"; "Mi terapeuta ni siquiera puede reunirse conmigo en persona". Los síntomas de COVID-19, especialmente la disnea, pueden agravar la ansiedad y los ataques de pánico que experimentan los pacientes. Esto puede conducir a dificultades para respirar, confundiendo dos orígenes para una oxigenación deficiente. La ansiedad puede llevar a ignorar los primeros síntomas del virus o a confabular los síntomas.

Las emergencias, las unidades psiquiátricas y los hospitales estatales podrían ver presentaciones psicóticas en personas con COVID-19 que necesitan tratamiento, reconociendo que estos síntomas con toda probabilidad no disminuirán cuando los síntomas de la infección se hayan resuelto. Estas personas necesitarán un seguimiento a largo plazo de sus síntomas psicóticos.

No es una sorpresa que la ansiedad tenga niveles altos durante la pandemia. Uno esperaría que las personas presenten síntomas de estrés postraumático.

En un estudio realizado en China, se observó que la tasa de ansiedad del personal médico era del 23,04\%, más altas en las mujeres que en los hombres y más alta en el personal de enfermería que en el cuerpo médicos. Del mismo modo, entre la población general china, el $53,8 \%$ tiene efectos psicológicos de moderados a graves; el $16,5 \%$ de síntomas depresivos moderados a graves, el $28,8 \%$ de síntomas de ansiedad y el $8,1 \%$ de estrés. (Lozano-Vargas, 2020, pág. 51)

En los trabajadores de la salud expuestos a COVID-19 en China, la depresión mostró una tasa mayor que cualquier síntoma, superando a la ansiedad y el insomnio. Al igual que con el estrés postraumático, algunos lograrán la resolución de esos síntomas a través de intervenciones breves, pero otros progresarán a un trastorno depresivo mayor y necesitarán un tratamiento a largo plazo. (Lozano-Vargas, 2020, pág. 51)

Además, más allá del miedo a la exposición o infección real por coronavirus que produce síntomas psiquiátricos, la cuarentena y aislamiento en sí induce síntomas psiquiátricos.

La cuarentena no solo exacerbará los síntomas en las personas con EMG conocida, sino que también puede llevar al tratamiento a las personas con EMG, que previamente no fueron diagnosticadas y / o no fueron tratadas debido a la exacerbación de los síntomas. (G. Druss, 2020)

Enfermedades afectadas por el aislamiento prolongado:

El aislamiento es un mecanismo de defensa por el cual las personas no olvidarán su trauma, sino que pierde su significado emocional al reprimirlo. Todas las enfermedades mentales se ven afectadas de diferentes formas por el aislamiento prolongado, pero en algunas en algunos trastornos, los efectos son más frecuentes:

Depresión paranoide: En la actualidad, esto corresponderá a la depresión en la que los síntomas de la psicosis y la emoción no son compatibles. Los delirios paranoicos aparecen en la depresión a través de tres posibles mecanismos:

1) Cuando el sujeto de la personalidad paranoide tiene signos de depresión leve, o cuando el sujeto con depresión leve es colocado en una situación que lo lleva a desarrolla un estado de paranoico (aislado no justificado, vergüenza, injusticia, etc.).

2) La aparición repentina de fotos de depresión, produciéndose "giro de la manecilla de la culpa" descrita por Sheyd, es un 
proceso que va desde la autocondena hasta el castigo a través del mecanismo de proyección. 3) Cuadros depresivos disfóricos, que se caracteriza por irritabilidad y puede causar paranoia.

Esquizofrenia residual: Esquizofrenia con inicio agudo de al menos un episodio, pero sin síntomas positivos o menos obvios en el momento del examen. En esta esquizofrenia predominan los síntomas negativos (emociones apagadas, apatía, ignorancia, aislamiento social, déficit de atención). Puede aparecer como un cuadro de transición agravada por una situación de gran estrés, como el aislamiento involuntario.

Trastorno esquizoide de la personalidad: Incluye las enfermedades más comúnmente encontradas en familiares no esquizofrénicos de pacientes con esquizofrenia, que se consideran expresión del mismo genotipo. Estos trastornos incluyen el aislamiento social, el consumo excesivo de alcohol, la personalidad paranoica, la personalidad abúlica, entre otros.

Demasiada información puede provocarnos estrés, ansiedad u angustia que afecta nuestro estado emocional, que nos lleva a realizar diversas comprobaciones, como buscar información nueva y actualizada. Para mitigar estos factores estresores y sus posibles consecuencias, existen algunas estrategias que pueden utilizarse para prevenir y reducir los efectos del aislamiento.

\section{ESTRATEGIAS PSICOLÓGICAS SUGERIDAS PARA SOBRELLEVAR LA CUARENTENA.}

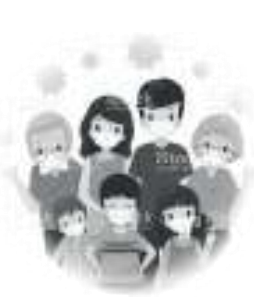

1. Aceptar la realidad y procurar hacer lo correcto

La situación que vivimos es tan complicada y es difícil comprender porque debemos quedarnos en casa, alejarnos de nuestros familiares y usar mascarilla cada vez que salimos a la calle.

Sin embargo, debemos de aceptar que "la realidad lamentablemente es la que es" y que es nuestra responsabilidad cumplir con las normas de higiene y distanciamiento social.

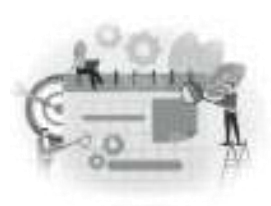

2. Planificar nuestro tiempo

Debido a la cuarentena nuestra vida ha empezado a sufrir varios cambios de manera sustancial. Por eso el mejor consejo es iniciar a planificar todo lo posible tanto en nuestro ambiente familiar como laboral para así sacar el mayor provecho a nuestro tiempo libre, siempre comprendiendo las necesidades específicas de los demás y respetando su espacio y tiempo.

Escriba un horario o lista de quehaceres que enumere las tareas que cada uno de los miembros de la familia debe realizar durante el día o la semana, y colóquelas donde todos puedan verlas.

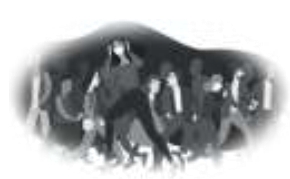

3. Evite la sobreinformación infoxicación

Es importante conocer los cambios diarios en la situación actual de nuestro país y el mundo. Sin embargo, debemos evitar la sobrecarga de información, a veces llamada "infoxicación” (ChamorroPremuzic, 2014) o “infobesidad” (Rogers, Puryear, \& Root, 2013). La sobre carga de información ocasiona dos resultados que son generalmente negativos.

Primero, es difícil distinguir entre datos reales y datos confirmados. Suelen difundirse a través de canales oficiales para distinguirlos de simples rumores o versiones no confirmadas.

El segundo efecto dañino es meterse en una espiral de malas noticias que en ocasiones son datos no confirmados, lo que solo conduce a la paranoia e inquietud. Se recomienda que evitemos hablar de estos temas todo el tiempo en especial, frente a los niños.

4. Mantente en contacto con tus seres queridos

Gracias a la tecnología, estar totalmente aislado y cuarentena no son sinónimos, porque hay una variedad de formas de comunicación como múltiples aplicaciones para escribir y hablar con otros, e incluso pueden interactuar sin importar que se encuentren en parte del mundo.

Además, incluso puedes hablar con tus vecinos entre balcones. Mantenerse en contacto y compartir información sobre sus condiciones de vida les brindará un gran apoyo y ayuda, y siempre siga el lema de no generar miedos y preocupaciones innecesarios, como se mencionó anteriormente. 

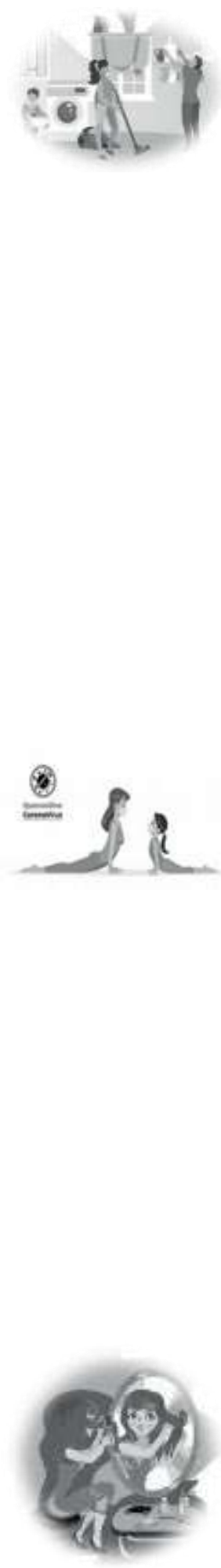

5. Aprovecha el tiempo en las cosas que antes no podías hacer

Debido a la absorción de nosotros por la escuela, el trabajo o la familia, casi todas las personas en algún momento de nuestras vidas estamos esperando realizar una determinada actividad o trabajo, por lo que este es el momento más adecuado para poder realizar todas estas actividades pendientes, como reparar la tubería que hace meses ha perdido de agua: Ya sea una persona que vive en familia o una persona que vive sola, hay muchos tipos de actividades que se pueden realizar. No solo pueden complacer y finalmente alcanzar estos objetivos, sino que también es una forma de pasar el tiempo más rápido y evitar de esta forma el aburrimiento de estar en casa.

\section{Haz ejercicio}

El hecho de que no podamos ir al gimnasio o hacer ejercicio al aire libre no significa que no podamos hacer ejercicio en casa. Existen algunas aplicaciones y tutoriales en Internet que pueden servir de guía para la práctica de deportes incluso en espacios reducidos. El resultado es puro beneficio: diviértete, evita los fenómenos fisiológicos sedentarios y mejora el estado de ánimo. Las endorfinas y otras hormonas secretadas por el cuerpo durante el ejercicio ayudan a reducir los síntomas de depresión y ansiedad, y contribuyen a la buena salud en general.

7. No descuides la salud y la higiene personal

Debido al aislamiento, debemos permanecer la mayor parte de nuestro tiempo en casa, por lo que podemos llevar ropa cómoda. Sin embargo, esto no significa que debamos olvidarnos de nuestra apariencia, el tiempo adecuado para dormir, una dieta equilibrada y no olvidarnos de tomar el sol al menos 20 minutos al día, bañarnos todos los días, peinarnos, cepillarnos los dientes y en caso de los hombres, afeitarnos la barba o bigote.

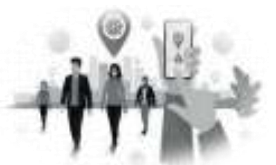

8. Estar atentos a los demás Debes concentrarte en las personas con las que vives. Por supuesto, no estar obsesionado, pero debes estar preparado para actuar como dicten las autoridades si hay situaciones preocupantes. También es importante cuidar tus pensamientos y emociones, prestando especial atención al contenido y las expresiones. Durante estos momentos difíciles, las emociones pueden romperse y la mala comunicación puede llevar a discusiones y momentos desagradables que solo causan daño.

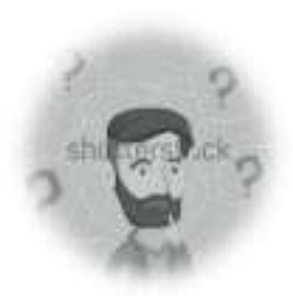

9. Trata de no pensar en periodos y fechas límite

"No fijes una fecha. Siempre debemos estar atentos, en algún momento todo volverá a la normalidad, y nunca debemos pensar que el tiempo es más corto de lo planeado.

Si crees que todo termina el 1 de junio y luego se extiende hasta el día 5 , esos cuatro días son un infierno. Al contrario, no sucederá. Si crees que has vuelto a la normalidad el día 5 y todo acaba el día 3, entonces esos días serán como un regalo.

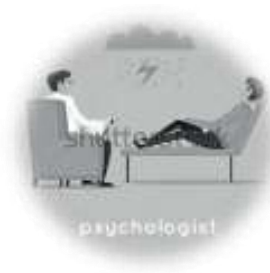

10. Si lo necesita, busque apoyo profesional

Aunque se ha enfatizado la importancia de no ignorar otros aspectos de la salud, la salud emocional aún requiere una atención especial.

Se trata de técnicas o estrategias para hacer frente a las restricciones que impone el aislamiento, que sin duda son de gran utilidad. Sin embargo, si alguien encuentra signos importantes de ansiedad en sí mismo o en las personas que viven con él, debe buscar ayuda de un psicólogo.

La salud mental y el bienestar son vitales para nuestra capacidad de pensar de manera colectiva y personal, expresar sentimientos, interactuar con los demás, ganarnos el sustento y disfrutar la vida. Sobre esta base, la 
promoción, protección y restauración de la salud mental puede considerarse una de las principales preocupaciones de las personas, las comunidades y las sociedades de todo el mundo.

\section{2.- BIBLIOGRAFÍAS}

Asensi, L. (S.F.). Cepsim Madrid. Obtenido De Https:// Www.Psicologiamadrid.Es/Blog/Articulos/ Psicología-Y-Coronavirus/Coronavirus-EfectosPsicologicos-Generados-Por-El-Confinamiento

Cdc. (30 De abril De 2020). Obtenido De Https:// Espanol.Cdc.Gov/Coronavirus/2019-Ncov/Daily-LifeCoping/Managing-Stress-Anxiety. Html

Chamorro-Premuzic, T. (13 De mayo De 2014). HowThe Web Distorts Reality And Impairs Our Judgement Skills. Obtenido De The Guardian: Https: //Www.Theguardian.Com/MediaNetwork/Media-Network-Blog/2014/May/13/ Internet-Confirmation-Bias

Daou, J. G. (14 De abril De 2020). Intra Med. Obtenido De Https: / /Www.Intramed.Net/ Contenidover .Asp?Contenidoid $=95946$

G. Druss, B. (3 De abril De 2020). Pandemia De Covid-19 En Poblaciones Con Enfermedades Mentales Graves. Obtenido De Especialidades Sld:Https: / Especialidades.Sld.Cu/ Psiquiatria/2020/04/09/Pandemia-De-Covid-19-EnPoblaciones-Con-Enfermedades-MentalesGraves/

Hajanian, T. (5 De abril De 2020). Semana. ObtenidoDe Https://Www.Semana.Es/Sociedad/ Coronavirus/Distanciamiento-SocialConsecuencias-Psicologicas-20200405-002197579/

Intra Med. (20 De abril De 2020). Obtenido De Https://Www.Intramed.Net/Contenidover. Asp?Contenidoid=95977

Intra Med. (2 De mayo De 2020). Obtenido De Https: / /Www.Intramed.Net/Contenidover. Asp?Contenidoid $=96056$

Lozano-Vargas, A. (2020). Impacto De La EpidemiaDel Coronavirus. Rev Neuropsiquiatr, 51. Obtenido De Http://Www.Scielo.Org.Pe/Pdf/Rnp/V83n1/16097394-Rnp-83-01-51.Pdf https://doi.org/10.20453/rnp.v83i1.3687

Organización Mundial De La Salud. (30 De Marzo De 2018). Obtenido De Https://W W W .Who.Int/Es/New S-R Oo M/F A Ct-Sheet S/ Detail/Mental-Health-S Treng Thening-Our Response
Rogers, P., Puryear, R., \& Root, J. (11 De junio De 2013). Infobesity: The Enemy Of Good Decisions.

Obtenido De Brief: Https://Www.Bain.Com/ Insight S/Inf Obesit Y - The-Enem Y - Of Good-Decisions

Samantha K Brooks, P. R. (12 De marzo De 2020). Intra Med. Obtenido De Https://Www.I N T R A M E D. NE T / C O N T E N I D O V E R . Asp?Contenidoid $=95688$

Unotv. (18 De marzo De 2020). Obtenido De Https:// Www.Unotv.Com/Noticias/Portal/Tecnologia/ Detalle/Coronavirus-Efectos-Psicologicos-DeLa-Cuarentena-Y-Como-Combatirlos-321880/

Vásquez, C. (17 De marzo De 2020). El Diario. Obtenido De Https://Www.Eldiario.Es/ Consum Ocl Ar O / Cuid Arse / Cor OnaVirus-Es Tra Tegias-Psicol Ogic As-Sobrellev ArCuarentena_0_1006500238.Html

\section{Sobre los autores}

\section{Rory René Vides Alonzo}

Es Profesor de la Carrera de Medicina y del sistema de estudios de Posgrado del Centro Universitario de Oriente CUNORI-USAC desde hace 13 años y egresado de la Universidad de San Carlos de Guatemala como Médico y Cirujano y de la Universidad Mariano Gálvez como Doctor en Psicología Médica y Salud Mental, con maestrías en Políticas y Estrategias para la Salud, Maestría en Psicología Médica y Salud Mental y Especialización en Investigación. Conferencista Nacional e Internacional (Panamá, Colombia y San Salvador) en temas de Inteligencia emocional e Investigador para la Dirección General de Investigación DIGI. Creador de dos maestrías. 1. Gerencia en Servicios de Salud y 2. Neuroclinica y Salud Mental. Creador de la especialización en Administración Hospitalaria y de Servicios de Salud. Investigador por pares para la Journal of Pharmaceutical Research International. Profesor de la Universidad autónoma de Santa Ana UNASA Santa Ana, San Salvador.

\section{Eileen Mariemilia Alegría Barrow}

Es Maestra de Educación Primaria, cursando actualmente la rotación de Salud Mental en 5to año de la Carrera de Médico y Cirujano en Centro Universitario de Oriente -CUNORI- Coautora del artículo de revista COVID19: Distanciamiento social, sus efectos psicológicos y 10 estrategias para sobrellevarlo. 


\section{Isolina Janeth Morales Guerra}

Es graduada de nivel medio como bachiller en Ciencias y Letras con orientación en computación, que cursa actualmente la rotación de salud mental, Quinto año de la carrera de médico y cirujano en el centro universitario de oriente -CUNORI-. Coautora del artículo de revista: COVID19: distanciamiento social, su efectos psicológicos y 10 estrategias para sobrellevarlo.

\section{Carlos Junior Sagastume Roque}

Es graduado de nivel medio como Bachiller en Ciencias y Letras, que cursa actualmente la rotación de salud mental, Quinto año de la carrera de Médico y Cirujano en el centro universitario de oriente -CUNORI-. Coautor del artículo de revista: COVID-19: Distanciamiento social, sus efectos psicológicos y 10 estrategias para sobrellevarlo.

\section{Juan Danilo Montejo Galdámez}

Es graduado de nivel media como bachiller industrial y perito en computación, que cursa actualmente la rotación de salud metal, 5to año de la carrera de médico y cirujano en el centro universitario de oriente -CUNORI-. Coautor del artículo de revista: COVID-19: distanciamientos sociales, su efectos psicológicos y 10 estrategias para sobrellevarlo.

Copyright (c) 2020 Rory Rene Vides Alonzo, Elie Mariemilia Alegría Barrow, Isolina Janeth Morales Guerra, Carlos Junior Sagastume Roque y Juan Danilo Montejo Galdámez

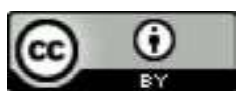

Este texto está protegido por una licencia Creative Commons 4.0.

Usted es libre para Compartir - copiar y redistribuir el material en cualquier medio o formato - y Adaptar eldocumento - remezclar, transformar y crear a partir del material- para cualquier propósito, incluso comercialmente, siempre que cumpla la condición de:

Atribución: Usted debe reconocer el crédito de una obra de manera adecuada, proporcionar un enlace a la licencia,e indicar si se han realizado cambios. Puede hacerlo en cualquier forma razonable, pero no de forma tal que sugieraque tiene el apoyo del licenciante o lo recibe por el uso que hace.

$\underline{\text { Resumen de licencia }}$ - Texto completo de la licencia 